\title{
Journal of Pediatric Neurology \&
}

\section{Paediatric Lenticulostriate Saccular Aneurysms-Challenges and Outcomes: A Case Report}

Sibhi Ganapathy ${ }^{1 *}$, Rajesh Nair ${ }^{2}$ and Girish Mehon

${ }^{1}$ Department of Neurosurgery, Manipal Hospitals, Karnataka, India

${ }^{2}$ Manipal University, Kasturba Medical College Manipal, Karnataka, India

*Corresponding author: Sibhi Ganapathy, Consultant, Department of Neurosurgery, Manipal Hospitals, Karnataka, India; Tel: 09686617831; E-mail: sibhig@yahoo.co.uk

Received date: August 03, 2018; Accepted date: September 16, 2018; Published date: September 23, 2018

Copyright: @ 2018 Ganapathy S, et al. This is an open-access article distributed under the terms of the Creative Commons Attribution License, which permits unrestricted use, distribution, and reproduction in any medium, provided the original author and source are credited.

\begin{abstract}
Distal lenticulostriate aneurysms are uncommon. They occur in a background of Moyamoya disease, vasculitis, collagen vascular disorders or AVMs. A prominent minority is idiopathic as well. Owing to the small caliber and high pressures seen in the lenticulostriate artery, dissections as well as fusiform dilatations are more common than saccular aneurysms. A common presentation of these aneurysms is a spontaneous basal ganglia hematoma with results in hemiplegia and dysphasia (depending upon the side of involvement) with or without obstructive hydrocephalus (due to the presence of intraventricular extension of the bleed.
\end{abstract}

Incidental detections of these aneurysms, especially in the pediatric age group are rare, as the diagnosis can only definitely be made on angiography. The detection is made difficult by the presence of concomitant pathologies, such as Moyamoya disease and vasculitis with obscure the imaging required for diagnosis.

We present the report of a leniculostriate saccular aneurysm, which presented with acute onset (15 mins) features of raised ICP associated with a hemiparesis. The aneurysm was surgically clipped. The patient had a transient post op hemiplegia, which completely recovered.

The report highlights the rarity of the disease, surgical challenges posed and the post op events to be expected and prevented in order for optimal recovery to occur.

Keywords: Vascular disorders; Lenticulostriate aneurysms; Stroke

\section{Introduction}

The lenticulostriate artery forms a crucial cog in the cerebrovascular juggernaut that supplies the brain. Despite its miniscule diameter and length, the artery is crucial as it supplies the internal capsule. Infarcts or hemorrhagic strokes of this artery are common owing to the small diameter of the artery and the high-pressure gradient experienced in it. Strokes usually result in a hemiplegia or paresis, which may or may not improve over time [1-3].

The most common cause for a lenticulostriate stroke is uncontrolled hypertension. The so-called "artery of Charcot" was described as early as 1823 as a leading cause of spontaneous intracerebral hemorrhage leading to hemiplegia [4].

An uncommon cause of lenticuostriate bleed is a distal aneurysm. Aneurysmal bleeds are rare [1]. Only about 36-40 cases have been reported in literature. Most aneurysms of the leniculostriate artery are fusiform or dissection related owing to the pressure gradient experienced by the artery [3]. Aneurysms are also seen in the backdrop of pathologies of the vascular tree such as Moya disease, Vasculitis or Collagen Vascular disease, which predispose the vascular wall to aneurysmal dilatation [2-4].

Due to the rare presentations as well as different nature and morphology of the aneurysms, management protocols are varied.
Endovascular stenting with or without coiling is described as superior to surgery in fusiform or dissecting aneurysms, but there is no consensus on saccular aneurysms [5].

There is an energetic discussion to treat incidental aneurysm with observation and intervene only if the child is symptomatic [5].

We present a case of a 12 yrs old child who presented with a saccular distal lenticulostriate aneurysm. We managed the aneurysm through a pterional craniotomy and microsurgical clipping of the aneurysm.

\section{Case Report}

A 12 yrs old male child, the only son of non-consanguineously married parents presented with sudden onset of weakness (progressing within 15 mins) of the left upper and lower limb with multiple episodes of vomiting and drowsy sensorium. There were no previous hospital admissions or any family history of vasculitis or recurrent strokes.

On examination he was found to be stable, obeying commands with normal speech, mild bradycardia with a $3 / 5$ weakness of the left upper and lower limbs. There were no neurocutaneous markers or cutaneous markers of vasculitis or SLE.

The child was immediately admitted in the ICU and stabilized. He was taken up for a CT brain, which showed a bleed in the basal ganglia region. 
Citation: Ganapathy S, Nair R, Menon G (2018) Paediatric Lenticulostriate Saccular Aneurysms-Challenges and Outcomes: A Case Report. J Pediatr Neurol Med 3: 132. doi:10.4172/2472-100X.1000132

Page 2 of 4

An angiogram done showed a saccular lenticulostriate aneurysm within the hematoma (Figures 1 \& 2) Blood work up for vasculitis and collagen vascular diseases were negative.

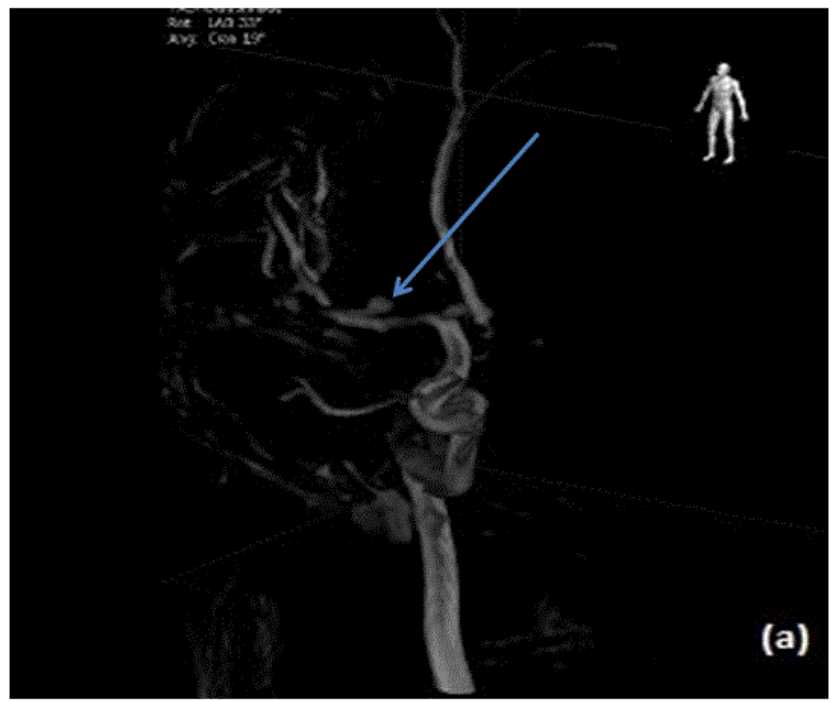

Figure 1: A 3D reconstructed image of the DSA of the right internal carotid system done showing the aneurysm in the lateral lenticulostriate arteries arising from the right MCA.

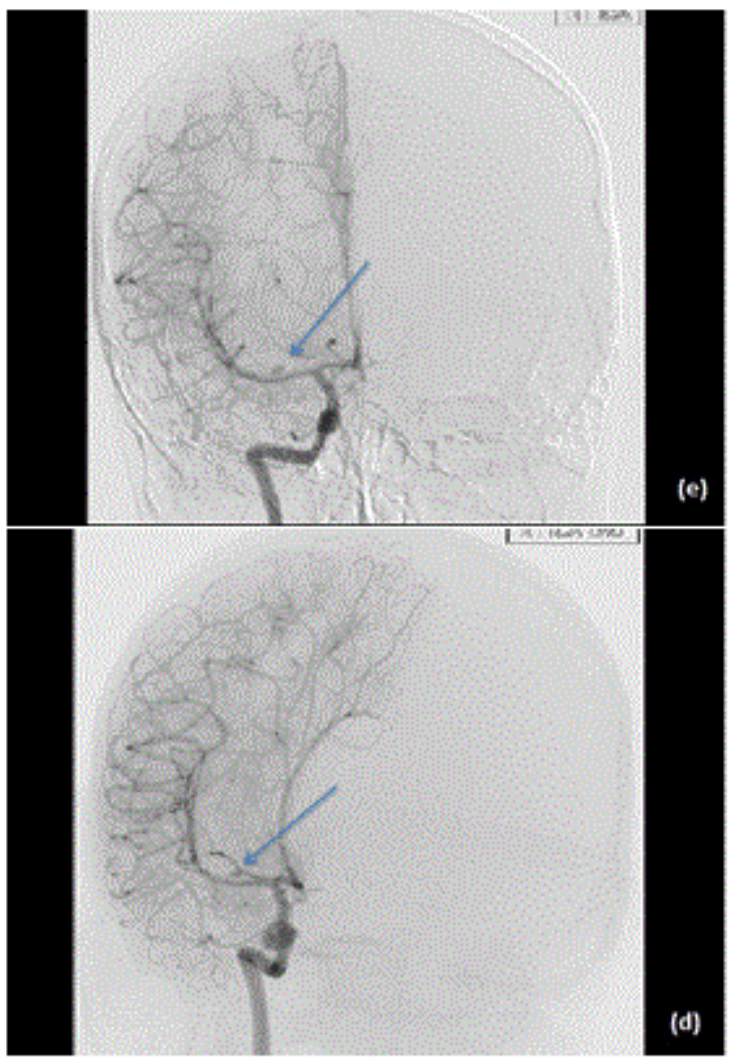

Figure 2: DSA of the child showing a small saccular lenticulostriate aneurysm as shown by the arrow.
Once stable, the child was taken up for a right pterional craniotomy and microsurgical clipping of the aneurysm. The surgery went well and the clip was so positioned as to prevent kinking of the vessel (Figure 3).

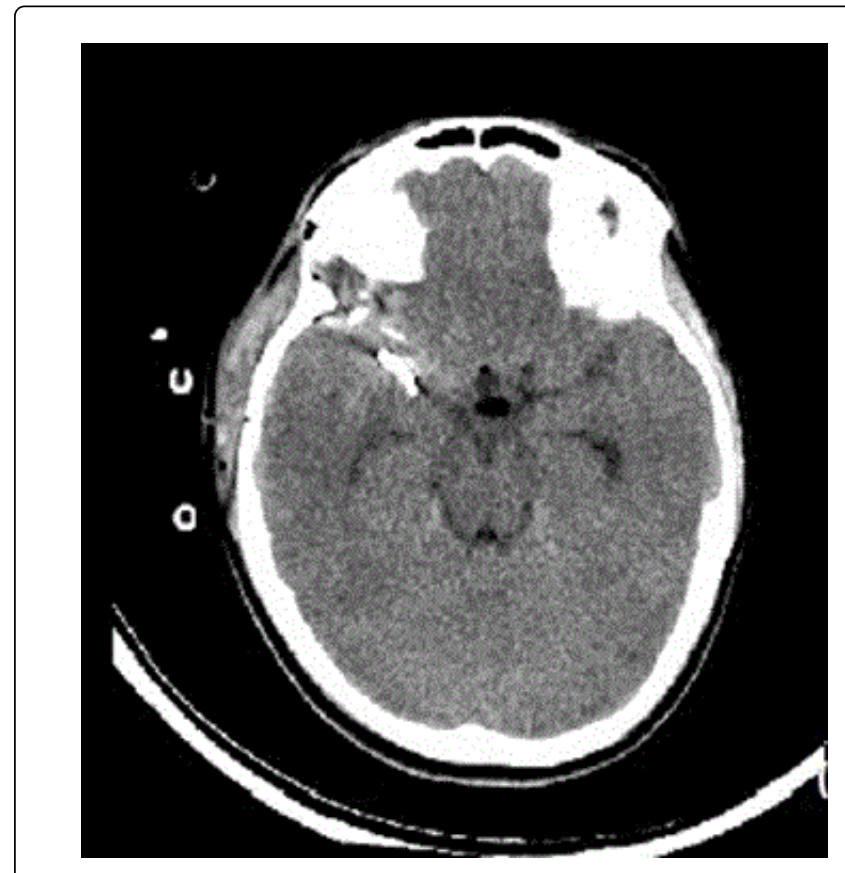

Figure 3: Ct brain of the patient on POD1 showing the clip in the right Sylvain fissure.

Post procedure the patient's sensorium improved well and he was shifted out of the ICU for physiotherapy and mobilization. His weakness however did not improve and gradually progressed into a complete plegia of both upper and lower limbs.

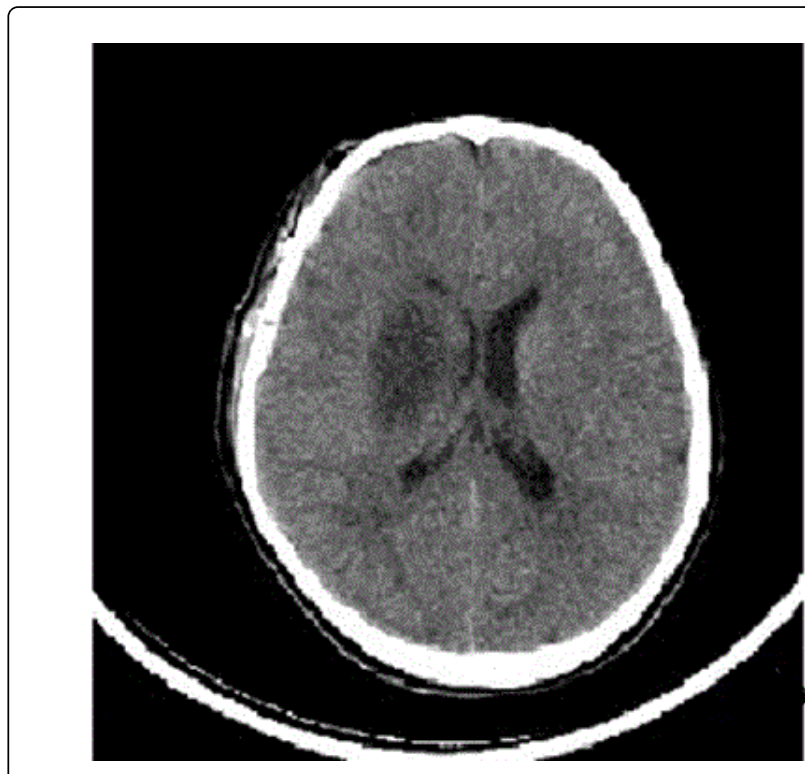

Figure 4: Delayed Post-Op CT of the child showing a right basal ganglia infarct. 
A repeat CT (done on POD 2) showed an infarct over the right basal ganglia and internal capsule region (Figure 4). The infarct was managed conservatively and the child was discharged to follow regular physiotherapy. The child was followed up regularly in OPD and showed steady improvement in power. 3 months later, the child was reviewed in OPD, and was found to have full power in both the left upper and lower limbs. A repeat angiogram showed no trace of the aneurysm, and the child now leads a normal and active life.

\section{Discussion}

As lenticulostriate aneurysms are so rare and so varied that a dependable protocol of management is not easy to establish. The presence of different etiologies as well as concomitant factors that contribute to the overall disease, make it a complex yet intriguing disease to treat.

In the above-mentioned case report, we experienced the following concerns:

\section{To intervene or not.}

A growing chorus of neurosurgeons worldwide suggest conservative watch and wait policy, as intervention maybe more traumatic and may be associated with more morbidity than observation. This is particularly clear in dissecting aneurysms as demonstrated by Leesien Yap and associates [6].

Our patient had a saccular aneurysm which both due to mass effect and rupture forced us to intervene surgically.

1. The eternal debate of endovascular intervention $v s$. open surgical clipping of the aneurysm.

Saccular aneurysms in the anterior circulation are more amenable to surgical clipping. Due to the small caliber of the artery and difficulty in cannulation, surgical intervention was considered in this patient [5].

\section{Post op Hemiparesis}

Care was taken intra-op to prevent kinking of the vessel due to the weight of the clip used, yet a delayed post op CT scan revealed an infarct over the right internal capsule and lentiform nucleus region. Causes of the infarct could range from kinking of the artery, spasm (although rare in children), occlusion of the vessel by thrombosis, or subclinical vasculitic processes that predisposed the child to spasm and thrombosis. The effects however were gradually lost and the child was seen on review walking and using his upper and lower limbs well with normal power $[4,5]$.

\section{The presence of concomitant/predisposing diseases}

Although the scans revealed and AVMs, Moya moya vessels and blood work showed no signs of SLE, Vasculitis or Infections, subclinical or early SLE maybe present and could have contributed to the disease. Subsequent tests as well as reviews with the local pediatrician were advised to detect changes early [2-4].

\section{Conclusion}

Lenticulostriate aneurysms are rare, yet an intriguing set of disorders with peculiar associations with systemic ailments. A high index of suspicion, careful technique and a good doctor patient relationship will result in good results. All options, from intervention to observation have shown results. Hence, a tailoring of the modality with the features of each patient with flexibility to change as per the situation is required to bring about the optimal outcome.

\section{References}

1. Mutsushito H, Amorim RL, Paiva WS, Cardeal DD, Pinto FC (2007) Idiopathic distal lenticulostriate artery aneurysm in a child. J Neurosurg 107: 419-424.

2. Kidoguchi J, Chiba M, Murakami T (1987) A case of systemic lupus erythematosus associated with an aneurysm of the lenticulostriate artery. No Shinkei Geka 15: 1221-1225.

3. Kuroda S, Houkin K, Kamiyama H (2001) Effects of surgical revascularization on peripheral artery aneurysms in moyamoya disease: Report of three cases. Neurosurgery 49: 463-467.

4. Murakami H, Mine T, Nakamura T (1984) Intracerebral hemorrhage due to rupture of true aneurysms of the lenticulostriate artery in moyamoya disease. Case report. Neurol Med Chir (Tokyo) 24: 794-799.

5. Eddleman CS, Surdell D, Pollock G (2007) Ruptured proximal lenticulostriate artery fusiform aneurysm presenting with subarachnoid hemorrhage: Case report. Neurosurgery 60: E949.

6. Yap L, Patankar T, Pysden K, Tyagi A, Goddard T (2015) Spontaneous dissecting lenticulostriate artery aneurysm in children: Radiologic findings and clinical management. J Child Neurol 30: 1060-1064. 